\title{
The Screening Value of Mammography for Breast Cancer: An Overview of 22 Systematic Reviews with Evidence Mapping
}

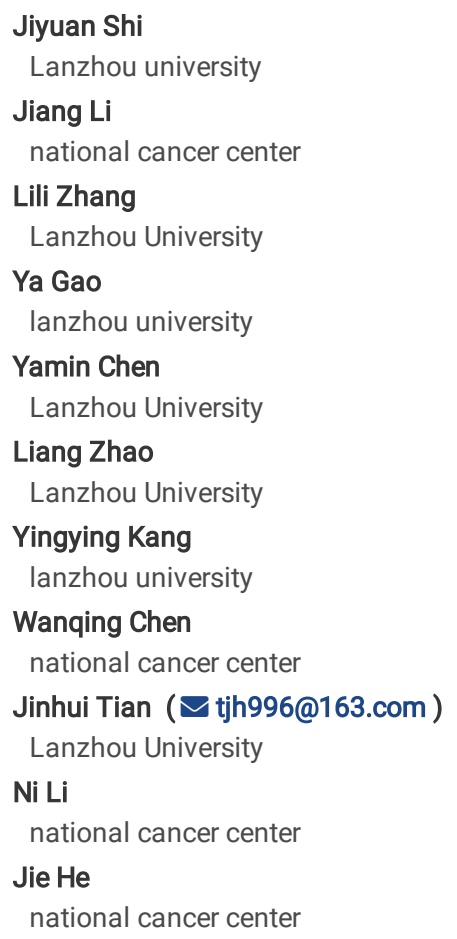




\section{Abstract}

Background: Several meta-analyses have evaluated the screening value of mammography for breast cancer, but the overall results have remained mixed or inconclusive.

Methods: Comprehensive literature search was conducted for SRs (systematic reviews) in Chinese Biomedical Databases (CBM), Cochrane Library, EMBASE, and PubMed until July 10, 2020. SRs with meta-analysis reported the benefit and performance of mammography screening were included. Two reviewers independently extracted data and performed the methodological quality assessments using The Risk Of Bias In Systematic Reviews (ROBIS). The characteristics of included SRs, the results of the quality of Risk of bias (RoBs) assessment and the pooled estimates of effect size were descriptively summarized using systematically structured tables and evidence mapping.

Results: Twenty two systematic reviews with meta-analysis were included. Only $13.6 \%$ of SRs were assessed as low-risk bias according to the overall risk of bias rating results in ROBIS tool. Pooled estimates for a reduction in breast cancer mortality attributable to mammography screening were range from 0.51 (OR, $95 \% \mathrm{Cl}: 0.46-0.55)$ to 1.04 (RR, $95 \% \mathrm{Cl}$ : 0.84-1.27). Sensitivity of difference mammography was ranged from $55 \%$ to $91 \%$, specificity of difference mammography was ranged from $84 \%$ to $97 \%$. According to the results of included SRs suggested, the statistically significant was observed that digital breast tomosynthesis (DBT) increased the cancer detected rate (CDR) and reduced the recall rate compared to digital mammography (DM), DM increased the CDR compared to screen-film mammography (SFM), and add DBT to digital or synthetic mammography increases the sensitivity, specificity, and CDR than DBT alone.

Conclusions: Further study should investigate the value of different imaging technology in breast cancer screening.

Systematic review registration: PROSPERO CRD42020207227

\section{Background}

Breast cancer (BC) is the most commonly diagnosed cancer and the leading cause of cancer death among women, nearly 2.1 million new cases of BC and 630000 cancer deaths owing to BC were recorded worldwide in 2018 [1]. Early detection of breast cancer through screening, effective diagnostic pathways, and optimal treatment have the ability to lower current breast cancer mortality rates and reduce the burden of this disease in the population. In the past few decades, mortality from BC has declined substantially [2]. Different screening tests have been used for implementing BC screening programmes among the general female population including breast self-examination, clinical breast examination (CBE), ultrasonography, and mammography [3]. Mammography has been recognized as an effective way to aid in the detection of $B C$ at an earlier stage, which may be associated with BC mortality reduction [4-6]. Numerous SRs have examined that screening with mammography reduces $\mathrm{BC}$ mortality among women, however, the result of $\mathrm{BC}$ mortality reduction was inconsistent and insufficient evidence was found for effectiveness varies by age [6-8].

Aiming to improve the performance of mammography screen BC, many new screen techniques have been used, such as digital mammography (DM), digital breast tomosynthesis (DBT), contrast-enhanced energy spectrum mammography (CESM), and DBT plus full-field digital mammography (FFDM). Recently, some SRs have compared the performance of different imaging techniques for BC screen, the overall results have remained mixed or inconclusive [9, 10], and the quality of these SRs has not been evaluated. Therefore, it is an indispensable step to comprehensively assessed the methodological quality of SRs before evidence-based recommendations can be determined.

The objective of the present overview of systematic reviews was: (i) to evaluate and map the quality of methodology of available SRs. (ii) to summarizes the existing evidence to determine the effectiveness of mammography screening in reducing BC mortality. (iii) to evaluate the evidence of the accuracy of different mammography techniques for BC screening.

\section{Materials And Methods}

\section{Study registration}

This overview has been registered on the international prospective register of systematic review (PROSPERO) (CRD42020207227). We reported this overview according to the preferred reporting items for a systematic review and meta-analysis of diagnostic test accuracy (PRISMA-DTA).

\section{Eligibility Criteria}

SRs with meta-analysis reported the value of mammography screening. (2) SRs must include women who were enrolled in BC screening programs without previously diagnosed BC. (3) SRs must report the value of screening mammography (association between mammography screening and breast cancer mortality reduction, sensitivity; specificity; cancer detection rate (CDR), area under curve (AUC), positive likelihood ratio (PLR), negative likelihood ratio (NLR), diagnostic odds ratio (DOR) and patient recall rate).

Studies were excluded if: (1) SRs did not focus on mammography screening such as diagnosis and recurrence. (2) narrative/methodological reviews, books, protocols, conference abstracts, letters, and SRs without meta-analysis.

\section{Search strategy}

We performed a systematic search in Chinese Biomedical Databases (CBM), Cochrane Library, EMBASE, and PubMed until July 10, 2020, to identify relevant SRs without language and publication status restrictions. The databases were searched using following search terms: "breast neoplasm", "phyllodes tumor", 
"intraductal carcinoma”, "lobular carcinoma”, "mammography”, "systematic review”, "meta-analysis”, and "meta-analysis". The search strategy of PubMed is available in Supplement.

\section{Study selection}

The identified records were imported into EndNote X8 (Thomson Reuters (Scientific) LLC Philadelphia, PA, US) for management. After the removal of duplicate records, the selection of potential reviews was performed first by titles and abstracts by two independent authors (JYS and LNX). The records that did not meet the inclusion criteria were excluded. The full text of each potential review was then obtained and assessed by the same two reviewers (JYS and YYK) to determine whether they meet the eligibility criteria. The discrepancies between the two authors were resolved by discussion with a third reviewer (JHT).

\section{Study selection and data extraction}

We used the EndNote X8 (Thomson Reuters (Scientific) LLC Philadelphia, PA, US) to manage Literature search records. Two independent reviewers (JYS, YYK) screened out possibly relevant studies by titles and abstracts extraction sheet to exclude records that did not meet the inclusion criteria. Then, the same two reviewers screen out the studies that met the inclusion criteria by evaluating the full text. Any disagreements were discussed and resolved by a third reviewer (JHT).

\section{Data extraction and management}

All authors involved in this study had previously piloted the form on a random sample of three included SRs to ensure the agreement among the interpretation of data items. One reviewer (JYS, and JL) extracted data from the included studies using a data extraction sheet, and a second reviewer (NL, and JHT) verified the extracted data. The extracted data included: authors, published year, country of the corresponding author, database searched, target population, intervention, intervention/exposure, comparison/control, outcome measures, study design, a methodological quality assessment tool used, number of included study and patients, follow up, the pooled results and their $95 \%$ confidence intervals $(\mathrm{Cl})$. Any disagreements were adjudicated by the third reviewer $(\mathrm{NL})$.

\section{Assessment of methodological quality}

The Risk Of Bias In Systematic Reviews (ROBIS) tool was recently published and aimed to help assessors judge the risk of bias (RoBs) in the review process, results, and conclusions that can be applied to SRs including both RCTs and non-RCTs [11, 12]. All reviewers involved in this study had previously piloted the form on a random sample of three included SRs to ensure the agreement among each criterion of ROBIS prior to employing this tool. Two review authors (J.Y.S, and H.J.) performed quality assessments independently using ROBIS, which is completed in three phases as follows: (1) Phase 1 assesses the relevance of the review and is considered optional; (2) Phase 2 includes four domains: study eligibility criteria, identification and selection of studies, data collection and study appraisal, synthesis, and findings; (3) Phase 3 assess the overall RoBs. The results of each domain and phase 3 were rated as "high risk", "low risk" or "unclear risk". Any disagreements were adjudicated by the third reviewer (J.H.T.).

\section{Data synthesis}

The pooled estimates of effect size from meta-analyses and their $95 \% \mathrm{Cl}$ were expressed as odds ratio (OR), relative risk (RR), Risk Difference (RD), or event rate (ER), depending on what the authors had reported. The characteristics of included SRs, the results of the quality of RoBs assessment and the pooled estimates of effect size were descriptively summarized using systematically structured tables and evidence mapping.

\section{Results}

\section{Search results}

The search of this overview retrieved 826 records, we excluded 191 articles after removing duplicates and screening title or abstract. The remaining 54 records, 9 were excluded due to the lack of target outcomes of this overview, 11 were excluded due to no meta-analysis conducted, 12 were excluded due to not focus on BC screening. Ultimately, 22 studies were included in this overview [2, 13-33]. Details of the PRISMA flow chart of literature studies for this overview are presented in Fig. 1.

\section{Characteristics of included SRs}

The details characteristics of the included 22 SRs with meta-analysis included primary studies ranging from 4 to 24 are presented in Table 1 . 19 from developed countries, 3 from developing countries, 17 SRs reported database searched, only 1 study retrieved Chinese databases. 11 assessed the methodological quality, 9 SRs used QUADAS-2, 1 SRs used QUADAS, 1 used quality score evaluation system for assessing the quality of RCTs published by Chalmers et al.

\section{Results of methodological quality}

ROBIS tool was evaluation was conducted in the 22 SRs. The assessment results of each item in phase 2 of the ROBIS tool are all presented in Table 2 , A total of 15 SRs (68.2\%) were assessed as having high RoBs on the domain 1. Domain 2 had $77.3 \%$ (17 SRs) of judgments as high RoBs. A total of 8 SRs (36.4 \%) were assessed to be at high RoBs on domain 3. Domain 4 had 59.1\% (13 SRs) of judgments as high RoBs. The final phase considered the overall RoBs, 19 SRs (86.4\%) were assessed to be high RoBs. The details of ROBIS assessment results are mapped in fig.2 and fig.3. 
Nine SRs were identified comparing the association of mammography vs. no screening on BC mortality. Pooled estimates for a reduction in BC mortality attributable to mammography screening stratified by study design were range from 0.51 (OR, $95 \% \mathrm{Cl}: 0.46-0.55)$ to 1.04 (RR, 95\% Cl: $0.84-1.27)$. An SRs of 10 case-control studies average a $49 \%$ reduction in BC mortality for women who are screened ( 0.51 [OR, $95 \%$ Cl: $0.46-0.55])$, which was similar to SRs with quasiRCTs conducted by Gøtzsche et al. (0.75 [RR, 95\% Cl: 0.67-0.83]), Hendrick et al. (0.71 [RR, 95\% Cl: 0.57-0.89] and Cochrane analysis (0.71 [RR, $95 \%$ Cl: $0.61-$ 0.83]) [26, 27, 32]. Pooled estimates of SRs with RCTs only conducted by Magnus (0.83 [RR, 95\% Cl: 0.72-0.97]), Gøtzschewere et al. (1.04 [RR, 95\% Cl: 0.84$1.27])$, and Cochrane analysis ( 0.93 [RR, $95 \% \mathrm{Cl}: 0.79-1.09])$ generally higher (mortality reduction lower) than with those observed with the other study design trials (Table 2) $[25,26,32]$.

In the SRs of RCTs that stratified by age, a significantly reduces BC mortality among women with the latest follow-up data (aged 40-49) invited to screening mammography was observed by Hendrick et al. (0.82[RR, 95\% Cl: 0.71-0.95], 7 RCTs) and Magnus et al. (0.83 [RR, 95\% Cl: 0.72-0.97)], 8 RCTs) [25, 27]; however, pooled estimates are not statistically significant for SRs conducted by Nelson et al. (0.92, [RR, 95\% Cl: 0.75-1.02], 9 trials), and Smart et al. (0.84 [RR, 95\% Cl: 0.69-1.02], 8 RCTs) [2,33]. The Cochrane analysis with adequately randomized trials found no significant BC mortality reduction among women below 50 years for follow up after 7 years $(0.94$ [RR, $95 \% \mathrm{Cl}$ : 0.78-1.14]) and 13 years (0.87 [RR, 95\% Cl: 0.73-1.03]). The Cochrane analysis reported that the pooled estimates for mortality reduction in screening women aged 50 years or older was lower when combined sub-optimally randomized trials with 7 and 13 years follow up (0.88 [RR, 95\% Cl: 0.64-1.20] and 0.94 [RR, 95\% Cl: 0.77-1.15]) than adequately randomized trials with 7 and 13 years (0.67 [RR, 95\% Cl: $0.56-0.81]$ and 0.70 [RR, 95\% Cl: 0.62-0.80]) [32]. An SRs conducted by Nelson et al. observed a statistically significant among women aged 50 to 59 (0.86 [RR, $95 \% \mathrm{Cl}$ : $0.68-0.97])$ and aged 60 to 69 years $(0.67$ [RR, 95\% Cl: 0.54-0.83]), the combined RR is not statistically significant for women aged 70-74 (P>0.05) [2].

\section{Performance of mammography screening technology}

Eight SRs reported the accuracy of BC screening conducted using mammography (Table 3). Sensitivity of difference mammography was ranged from $55 \%$ to $90.77 \%$, specificity of difference mammography was ranged from $84 \%-97 \%$. The sensitivity of DBT + FFDM, CESM, DM, FM, and FFDM, were $90.77 \%(95 \%$ Cl: 80.7\%-96.51\%), 89\%(95\% Cl: 88\%-91\%), 76\%(95\% Cl: 70\%-81\%), 76\% (95\% Cl: 70\%-81\%), and 60.00\% (95\% Cl, $47.10 \%-71.96 \%)$, respectively. The specificity of FM, DBT + FFDM, DM, FFDM, and CESM were 97\% (95\% Cl: 94\%-98\%), 96.49\% (95\% Cl: 96.04\%-96.90\%), 96\% (95\% Cl: 94\%-97\%), 95.55\% (95\% Cl: 95.04\%-96.01\%), and 84\% (95\% Cl: 82\%-85\%). The DOR of BC screening conducted using the CESM, DM, SFM were 71.36(95\% Cl: 36.28-140.39), 72(95\% Cl: 44-118), and 91(95\% Cl: 52-157).

Seven SRs compared the performance of difference mammography (Table 4). The pooled estimates for CDR of DBT vs. DM, DBT + SM vs. DM, and DBT + FFDM vs. FFDM were 0.0016 (RD, 95\% Cl: 0.0011-0.002), 1.38(RR, 95\% Cl:1.24-1.54), and 1.29 (RR, 95\% Cl: 1.164-1.429). The CDR of FM was similar to FFDM (0.93[RR, 95\% Cl: 0.83-1.03]), two studies evaluated the CDR of DBT + DM and DM and statistically significant were both observed (1.36 [RR, 95\% Cl: 1.18-1.58] and 1.52 [RR, 95\% Cl: 1.08-2.12]). Moreover, the pooled estimates for CDR of DM vs. FM in two SRs found a statistically significant (1.17 [RR, $95 \%$ Cl: $1.06-$ 1.29] and 0.00051 [RD, $95 \% \mathrm{Cl}: 0.00019-0.00083])$. The risk ratios and the respective $95 \% \mathrm{Cl}$ of recall rate of DM vs. FM in two SRs were inconsistency (1.07 [RR, 95\% Cl: 0.94-1.22] and 0.00695 [RD, 95\% Cl: 0.00347-0.01042]). Moreover, the risk ratios and the respective $95 \% \mathrm{Cl}$ of recall rate of DBT + DM vs. DM were also inconsistency (1.13 [RR, 95\% Cl: 0.96-1.32] and 0.72[RR, 95\% Cl: 0.64-0.80]). The recall rate of FM vs. FFDM and DBT+SM vs. DM were not statistically significant (0.95 [RR, 95\% Cl: 0.71-1.26] and 1.08 [RR, 95\% Cl: 0.92-1.26]). The RD of recall rate of DBT was statistically significant lower than DM (-0.0219 $[-0.0298,-0.014])$.

\section{Discussion}

To our knowledge, this overview is the first one to systematically evaluate the methodological quality of evidence from SRs to provide an evidence-based assessment and summarize the accuracy and efficacy on BC screen. A comprehensive literature search was performed and 22 SRs were identified, published from 1995 to 2020, the number of studies included in each SRs ranged from 4 to 24.

\section{Risk of bias in systematic reviews}

High standards SRs can provide the highest level of evidence for evidence-based decision making, while low-quality design or conduct of SRs have the potential to bias results and mislead clinical practice. It is crucial to assess the quality of SRs before being served a vital role in decision making. We performed the ROBIS tool to assess the quality of included SRs and the results were not optimistic. None of the SRs met all four domains in phase 2. Only $36.4 \%$ of SRs with low RoBs in study eligibility criteria, SRs without prior protocol may reduce the transparency and increase the RoBs in researcher outcome reporting, previous studies discovered that the quality of SRs could be improved if they performed a priori protocol. However, only six SRs reported prior study protocol or registration, which is similar to previous studies. Although some studies were clearly described question, objective, and criteria, it was still difficult to judgment about whether these SRs were stipulated in advance and avoid the RoBs. Only $18.2 \%$ with low risk for each signaling questions in domain 2 (identification and selection of studies), thirteen SRs searched three or more databases, six of the studies reported detailed search strategy, identified unpublished studies, and searched registry platform, eight studies did not report the follow-up time, seven studies did not involve at least two reviewers independently performing the process of selecting studies for inclusion. $45.4 \%$ of SRs with low risk for domain 3 (data collection and study appraisal), $50 \%$ of SRs assessed the RoBs or methodological quality using the appropriate tool, and only eight studies assessed the RoBs of included SRs independently by at least two reviewers. $59.1 \%$ of SRs had potential methodological flaws in synthesis and findings according to domain 4 . First, the inadequate search of existing literature may ignore the results of some studies. Second, the lack of a bias risk assessment process and the inclusion of primary studies with bias may result in biased and unreliable results. Third, it is difficult to determine whether the data synthesis and analysis methods have been determined in advance and have been followed due to the lack of protocol and registration information. Last, substantial heterogeneity was observed, while further analyses were not conducted to explore the source of the variation. Only $13.6 \%$ of SRs were assessed as low-risk bias according to the overall RoBs rating results. 
Nine SRs explored the effectiveness of mammography screening, pooled estimates for the reduction in BC mortality were ranging from 0.51 to 1.04 . SRs included RCT found a lower BC mortality than SRs included other study designs, which was similar to the sub-group analysis of Cochrane analysis. In the SRs that were stratified by age, the result of three SRs of RCTs found a statistically significant reduction in mortality among women ages 40 to 49 years with screening mammography, while pooled estimates are not statistically significant for women aged 40 to 49 years in other two SRs. According to Cochrane analysis, the subgroup of SRs only combined sub-optimally adequately randomized found a statistically significant reduction in mortality in women younger than 50 years old [32]. SRs conducted by Nelson et al. found BC mortality is generally reduced with screening among women aged 50-59 and 60-69 [2], however, pooled estimates are not statistically significant for women aged 70 to 74 years. Both Kerlikowske et al. and Cochrane's analysis found that screening mammography significantly reduces BC mortality in women aged 50 to 74 years, while Cochrane analysis found no statistically significant only combine adequately randomized trials [28, 32]. In general, most of the included SRs suggested that mammography screening was a benefit to BC mortality reduction, while results were not statistically significant at all SRs. Moreover, the benefit of mammography screening for BC mortality reduction among women aged 40 to 49 was contradictory between included SRs.

\section{Performance of mammography screening technology}

Improvements in mammographic technology provided the transition from FM to DM and DBT, which may increase the benefit of screening by increasing the detection accuracy of BCs. Five studies reported the sensitivity and specificity of mammography ranged from $55 \%$ to $90.77 \%$ and $84 \%$ to $97 \%$. Hodgson et al. found DBT plus FFDM has higher sensitivity and specificity than FFDM alone, and Song et al. observed that the performance of DM was similar to that of FM $[17,20]$. Zhu et al. found that CESM has high diagnostic accuracy, while significant heterogeneity was observed and no potential source of heterogeneity could analysis due to partial loss of data.

Six studies compared the CDR and recall rate of different mammography, both of SRs conduct by lared et al. and Farber et al. found the cancer detection rate of DM was statistically significantly higher than FM, however, the recall rate was not significant differences in former, but significant differences increased by later $[14,23]$. Vinnicombe et al. declared that there was no evidence of differences between FFDM and FM in CDR and recall rate [15]. Marinovich et al. found a statistically significant improves CDR and reduces recall for DBT vs. DM [21]. The use of DBT plus digital or synthetic mammography could improve the CDR of $\mathrm{BC}$, while the recall rate was observed reduction by phi et al. and no significant improvement by Giampietro et al. [16, 22]. Yun et al. found that adding DBT to FFDM is more effective in detecting BC than FFDM alone, especially in increasing the detection of early invasive BC specificity, and CDR than DBT alone [19]. According to the results of included SRs, most of the included reviews suggested that the CBT increased the CDR and reduced the recall rate compared to DM, DM increased the CDR compared to FM, and add DBT to digital or synthetic mammography increases the sensitivity, specificity, and CDR than DBT alone. However, the potential methodological flaw and different reference standards challenge the interpretation of the results. More high-quality SRs and primary studies are needed to comprehensively determine the performance of difference mammography.

\section{Strengths and limitations}

To our knowledge, this is the first overview to investigate the methodological quality of the SRs using the ROBIS tool and assess the benefit of mammography screening in BC mortality reduction and accuracy of mammography. The present overview has some limitations: First, SRs were included in 1995 and 2020 , the result of mortality reduction of $\mathrm{BC}$ may be increased due to the improved treatment of more advanced cancer and $\mathrm{BC}$ awareness, and the decision to participate in screening programs should trade-offs carefully considering combined the over-diagnosis, costs and other influence. Second, both Chinese and English databases were searched, but there were only English language studies met our criteria, which may exist bias of language. Finally, a high RoBs of the included SRs might affect the reliability of the evidence summarized.

\section{Conclusion}

Most of the included reviews concluded that breast cancer mortality is generally reduced with mammography screening, with the exception of women under the age of 50 and those aged 70 to 74 . With the development of advanced imaging technology, the diagnostic performance of mammography has been improving, most of the included SRs suggested that DM increased the CDR compared to FM, the CBT increased the CDR and reduced the recall rate compared to DM, moreover, add DBT to digital or synthetic mammography increases the sensitivity, specificity, and CDR than DBT alone. However, the methodological quality of most of the included reviews was defined as high risk, with the publication of methodological tool, as well as reporting checklist, improvement is urgent need over time.

\section{Abbreviations}

BC Breast cancer;

DM digital mammography;

CESM Contrast-Enhanced Spectral Mammography;

DBT digital breast tomosynthesis;

FM film mammography;

FFDM full-field digital mammography;

CBM Chinese Biomedical Databases; 
ROBIS The Risk Of Bias In Systematic Reviews;

DOR diagnostic odds ratio; CDR, cancer detection rate;

AUC area under curve; PPV positive predictive value;

FPR false-positive rate;

NLR negative likelihood ratio;

RCT randomised controlled trial.

\section{Declarations}

\section{Acknowledgments}

Thanks to the National Cancer Center and the Evidence-based Medicine Center Of Lanzhou University for their support. Thanks to Dr. Long Ge provided methodology guidance.

\section{Funding}

This study was supported by the grants from the Sanming Project of Medicine in Shenzhen (SZSM201911015) and Training Programme Foundation for the Talents in Beijing City Grant (No.2017000021223TD05).

\section{Contributions}

All authors made a substantial contribution to the research. JHT, NL and JH: conceptualization. YYK, LNX, JL and JYS: collected and assayed the data.. All authors: writing-review \& editing. JHT, NL and JH: revised.

\section{Ethics declarations}

\section{Ethics approval and consent to participate}

Ethical approval and patient consent are not required since this is an overview based on published studies.

\section{Consent for publication}

Not applicable.

\section{Competing interests}

The authors declare that the research was conducted in the absence of any commercial or financial relationships that could be construed as a potential conflict of interest.

\section{Informed consent}

All analyses were based on previously published studies; thus, no informed consent is required.

\section{References}

1. Bray F, Ferlay J, Soerjomataram I, Siegel RL, Torre LA, Jemal A. Global cancer statistics 2018: GLOBOCAN estimates of incidence and mortality worldwide for 36 cancers in 185 countries. CA Cancer J Clin. 2018 Nov;68(6):394-424. doi: 10.3322/caac.21492.

2. Nelson HD, Fu R, Cantor A, Pappas M, Daeges M, Humphrey L. Effectiveness of Breast Cancer Screening: Systematic Review and Meta-analysis to Update the 2009 U.S. Preventive Services Task Force Recommendation. Ann Intern Med. 2016 Feb 16;164(4):244-55. doi: 10.7326/M15-0969.

3. Yang L, Wang S, Zhang L, et al. Performance of ultrasonography screening for breast cancer: a systematic review and meta-analysis. BMC Cancer. 2020 Jun 1;20(1):499. doi: 10.1186/s12885-020-06992-1.

4. DeSantis C, Ma J, Bryan L, Jemal A. Breast cancer statistics, 2013. CA Cancer J Clin. 2014 Jan-Feb;64(1):52-62. doi: 10.3322/caac.21203.

5. DeSantis C, Siegel R, Jemal A. Breast cancer statistics, 2011. CA Cancer J Clin. 2011 Nov-Dec;61(6):409-18. doi: 10.3322/caac.20134. Epub 2011 Oct 3. PMID: 21969133

6. Myers ER, Moorman P, Gierisch JM, et al. Benefits and Harms of Breast Cancer Screening: A Systematic Review. JAMA. 2015 Oct 20;314(15):1615-34. doi: 10.1001/jama.2015.13183.

7. Brodersen J, Jørgensen KJ, Gøtzsche PC. The benefits and harms of screening for cancer with a focus on breast screening. Pol Arch Med Wewn. 2010 Mar;120(3):89-94.

8. Krager SC, Prochazka AV. ACP Journal Club. Review: In women $\geq 40$ years of age at average risk, breast cancer screening causes some harms. Ann Intern Med. 2016 Apr 19;164(8):JC39. doi: 10.7326/ACPJC-2016-164-8-039. 
9. Nothacker M, Duda V, Hahn M, et al. Early detection of breast cancer: benefits and risks of supplemental breast ultrasound in asymptomatic women with mammographically dense breast tissue. A systematic review. BMC Cancer. 2009 Sep 20;9:335. doi: 10.1186/1471-2407-9-335.

10. Movik E, Dalsbø TK, Fagelund BC, Friberg EG, Håheim LL, Skår Å. Digital Breast Tomosynthesis with Hologic 3D Mammography Selenia Dimensions System for Use in Breast Cancer Screening: A Single Technology Assessment [Internet]. Oslo, Norway: Knowledge Centre for the Health Services at The Norwegian Institute of Public Health (NIPH); 2017 Sep 4. Report from the Norwegian Institute of Public Health No. 2017-08.

11. Perry R, Leach V, Davies P, Penfold C, Ness A, Churchill R. An overview of systematic reviews of complementary and alternative therapies for fibromyalgia using both AMSTAR and ROBIS as quality assessment tools. Syst Rev. 2017 May 15;6(1):97. doi: 10.1186/s13643-017-0487-6.

12. Whiting P, Savović J, Higgins JP, et al. ROBIS group. ROBIS: A new tool to assess risk of bias in systematic reviews was developed. J Clin Epidemiol. 2016 Jan;69:225-34. doi: 10.1016/j.jclinepi.2015.06.005.

13. Zhu X, Huang JM, Zhang K, et al. Diagnostic Value of Contrast-Enhanced Spectral Mammography for Screening Breast Cancer: Systematic Review and Meta-analysis. Clin Breast Cancer. 2018 Oct;18(5):e985-e995. doi: 10.1016/j.clbc.2018.06.003.

14. lared W, Shigueoka DC, Torloni MR, et al. Comparative evaluation of digital mammography and film mammography: systematic review and metaanalysis. Sao Paulo Med J. 2011;129(4):250-60. doi: 10.1590/s1516-31802011000400009.

15. Vinnicombe S, Pinto Pereira SM, McCormack VA, et al. Full-field digital versus screen-film mammography: comparison within the UK breast screening program and systematic review of published data. Radiology. 2009 May;251(2):347-58. doi: 10.1148/radiol.2512081235.

16. Giampietro RR, Cabral MVG, Lima SAM, et al. Accuracy and Effectiveness of Mammography versus Mammography and Tomosynthesis for PopulationBased Breast Cancer Screening: A Systematic Review and Meta-Analysis. Sci Rep. 2020 May 14;10(1):7991. doi: 10.1038/s41598-020-64802-x.

17. Hodgson R, Heywang-Köbrunner SH, Harvey SC, et al. Systematic review of 3D mammography for breast cancer screening. Breast. 2016 Jun;27:52-61. doi: 10.1016/j.breast.2016.01.002.

18. Souza FH, Rosa MI, Polanczyk CA. Is full-field digital mammography more accurate than screen-film mammography in overall population screening? A systematic review and meta-analysis. Breast. 2013 Jun;22(3):217-24. doi: 10.1016/j.breast.2013.02.013.

19. Yun SJ, Ryu CW, Rhee SJ, Ryu JK, Oh JY. Benefit of adding digital breast tomosynthesis to digital mammography for breast cancer screening focused on cancer characteristics: a meta-analysis. Breast Cancer Res Treat. 2017 Aug; 164(3):557-569. doi: 10.1007/s10549-017-4298-1.

20. Song SY, Park B, Hong S, Kim MJ, Lee EH, Jun JK. Comparison of Digital and Screen-Film Mammography for Breast-Cancer Screening: A Systematic Review and Meta-Analysis. J Breast Cancer. 2019 May 13;22(2):311-325. doi: 10.4048/jbc.2019.22.e24.

21. Marinovich ML, Hunter KE, Macaskill P, Houssami N. Breast Cancer Screening Using Tomosynthesis or Mammography: A Meta-analysis of Cancer Detection and Recall. J Natl Cancer Inst. 2018 Sep 1;110(9):942-949. doi: 10.1093/jnci/djy121.

22. Phi XA, Tagliafico A, Houssami N, Greuter MJW, de Bock GH. Digital breast tomosynthesis for breast cancer screening and diagnosis in women with dense breasts - a systematic review and meta-analysis. BMC Cancer. 2018 Apr 3;18(1):380. doi: 10.1186/s12885-018-4263-3.

23. Farber R, Houssami N, Wortley S, et al. Impact of Full-Field Digital Mammography versus Film-Screen Mammography in Population Screening: a Metaanalysis. J Natl Cancer Inst. 2020 Jun 23:djaa080. doi: 10.1093/jnci/djaa080.

24. Nickson C, Mason KE, English DR, Kavanagh AM. Mammographic screening and breast cancer mortality: a case-control study and meta-analysis. Cancer Epidemiol Biomarkers Prev. 2012 Sep;21(9):1479-88. doi: 10.1158/1055-9965.

25. Magnus MC, Ping M, Shen MM, Bourgeois J, Magnus JH. Effectiveness of mammography screening in reducing breast cancer mortality in women aged 39-49 years: a meta-analysis. J Womens Health (Larchmt). 2011 Jun;20(6):845-52. doi: 10.1089/jwh.2010.2098.

26. Gøtzsche PC, Olsen O. Is screening for breast cancer with mammography justifiable? Lancet. 2000 Jan 8;355(9198):129-34. doi: 10.1016/S01406736(99)06065-1.

27. Hendrick RE, Smith RA, Rutledge JH, Smart CR. Benefit of screening mammography in women aged 40-49: a new meta-analysis of randomized controlled trials. J Natl Cancer Inst Monogr. 1997;(22):87-92. doi: 10.1093/jncimono/1997.22.87.

28. Kerlikowske K, Grady D, Rubin SM, Sandrock C, Ernster VL. Efficacy of screening mammography. A meta-analysis. JAMA. 1995 Jan 11;273(2):149-54.

29. Jacklyn G, Glasziou P, Macaskill P, Barratt A. Meta-analysis of breast cancer mortality benefit and overdiagnosis adjusted for adherence: improving information on the effects of attending screening mammography. Br J Cancer. 2016 May 24;114(11):1269-76. doi: 10.1038/bjc.2016.90.

30. Posso M, Puig T, Carles M, Rué M, Canelo-Aybar C, Bonfill X. Effectiveness and cost-effectiveness of double reading in digital mammography screening: A systematic review and meta-analysis. Eur J Radiol. 2017 Nov;96:40-49. doi: 10.1016/j.ejrad.2017.09.013.

31. Phi XA, Houssami N, Hooning MJ, et al. Accuracy of screening women at familial risk of breast cancer without a known gene mutation: Individual patient data meta-analysis. Eur J Cancer. 2017 Nov;85:31-38. doi: 10.1016/j.ejca.2017.07.055.

32. Gøtzsche PC, Jørgensen KJ. Screening for breast cancer with mammography. Cochrane Database Syst Rev. 2013 Jun 4;2013(6):CD001877. doi: 10.1002/14651858.CD001877.pub5.

33. Smart CR, Hendrick RE, Rutledge JH 3rd, Smith RA. Benefit of mammography screening in women ages 40 to 49 years. Current evidence from randomized controlled trials. Cancer. 1995 Apr 1;75(7):1619-26. doi: 10.1002/1097-0142(19950401)75:7<1619::aid-cncr2820750711>3.0.co;2-t

\section{Tables}

Due to technical limitations, table 1 is only available as a download in the Supplemental Files section. 
Table 2 . Main results of the included meta-analysis evaluating association between mammography screening and breast cancer mortality

\begin{tabular}{|c|c|c|c|c|c|}
\hline Study & Age & Study design & Participates & Follow up & $\mathrm{ES}(95 \% \mathrm{Cl})$ \\
\hline Nickson(2012) & $40-75$ & $\operatorname{ccs}(10)$ & NR & NR & OR:0.51(95\% Cl 0.46-0.55) \\
\hline Magnus(2011) & $39-49$ & $\operatorname{RCTs}(7)$ & 336261 & 10-18 years & RR:0.83(95\% Cl 0.72-0.97) \\
\hline \multirow[t]{2}{*}{ Gøtzsche(2000) } & $39-59$ & $\operatorname{RCTs}(2)$ & 132118 & 9-12 year & RR:1.04(95\% Cl 0.84-1.27) \\
\hline & & quasi-RCTs(6) & 324231 & 9-12 year & RR:0.75(95\% Cl 0.67-0.83) \\
\hline Hendrick(1997) & $40-49$ & $\operatorname{RCTs}(8)$ & 211150 & 12.7 years(rang from 10.5 to 18 ) & RR:0.82(95\% Cl 0.71-0.95) \\
\hline \multirow[t]{9}{*}{ Kerlikowske(1995) } & $40-49$ & $\operatorname{RCTs}(8) ; \operatorname{CCS}(1)$ & NR & $7-12$ years & RR:0.93(95\% Cl 0.76-1.13) \\
\hline & & $\operatorname{RCTs}(8) ; \operatorname{CcS}(1)$ & NR & 7-9 years & RR:1.02(95\% Cl 0.82-1.27) \\
\hline & & $\operatorname{RCTs}(8) ; \operatorname{CCS}(1)$ & NR & 10-12 years & RR:0.83(95\% Cl 0.65-1.06) \\
\hline & $40-74$ & $\operatorname{RCTs}(8) ; \operatorname{CcS}(4)$ & NR & $7-12$ years & RR:0.75(95\% Cl 0.68-0.83) \\
\hline & & $\operatorname{RCTs}(8) ; \operatorname{CCS}(5)$ & NR & 7-9 years & RR:0.78(95\% Cl 0.69-0.89) \\
\hline & & $\operatorname{RCTs}(8) ; \operatorname{CcS}(6)$ & NR & 10-12 years & RR:0.77(95\% Cl 0.68-0.86) \\
\hline & $50-74$ & $\operatorname{RCTs}(8) ; \operatorname{ccs}(2)$ & NR & $7-12$ years & RR:0.74(95\% Cl 0.66-0.83) \\
\hline & & $\operatorname{RCTs}(8) ; \operatorname{CcS}(2)$ & NR & 7-9 years & RR:0.73(95\% Cl 0.63-0.84) \\
\hline & & $\operatorname{RCTs}(8) ; \operatorname{CCS}(2)$ & NR & 10-12 years & RR:0.76(95\% Cl 0.67-0.87) \\
\hline Jacklyn(2016) & $39-75$ & $\operatorname{RCTs}(9)$ & 599090 & 13 years & RR:0.78 (95\% Cl 0.72-0.85) \\
\hline \multirow[t]{24}{*}{ Gøtzsche (2013) } & $39-74$ & ART囚4囚 & 616327 & 7 years & RR:0.81(95\% Cl 0.72-0.90) \\
\hline & & SRT囚7囚 & & & \\
\hline & & ART $\triangle 4 \bigotimes$ & 292958 & 7 years & RR:0.93(95\% Cl 0.79-1.09) \\
\hline & & SRT囚7凹 & 323369 & 7 years & RR:0.71(95\% Cl 0.61-0.83) \\
\hline & & ART $₫ 4 \rrbracket$ & 599090 & 13 years & RR:0.81(95\% Cl 0.74-0.87) \\
\hline & & SRT $₫ 5 \square$ & & & \\
\hline & & ART囚4囚 & 292153 & 13 years & RR:0.90(95\% Cl 0.79-1.02) \\
\hline & & SRT $₫ 5 \rrbracket$ & 306937 & 13 years & RR:0.75(95\% Cl 0.67-0.83) \\
\hline & $\otimes 50$ & ART $₫ 3 \bigotimes$ & 356368 & 7 years & RR:0.89(95\% Cl 0.77-1.04) \\
\hline & & SRT囚5囚 & & & \\
\hline & & ART $₫ 3 \otimes$ & 227333 & 7 years & RR:0.94(95\% Cl 0.78-1.14) \\
\hline & & SRT $₫ 5 \rrbracket$ & 129035 & 7 years & RR:0.81(95\% Cl 0.63-1.05) \\
\hline & & ART囚3囚 & 329511 & 13 years & RR:0.84(95\% Cl 0.73-0.96) \\
\hline & & SRT $₫ 5 \rrbracket$ & & & \\
\hline & & ART囚3囚 & 218697 & 13 years & RR:0.87(95\% Cl 0.73-1.03) \\
\hline & & SRT $₫ 5 \bigotimes$ & 110814 & 13 years & RR:0.80(95\% Cl 0.64-0.98) \\
\hline & $\geq 50$ & ART $₫ 2 \bigotimes$ & 261044 & 7 years & RR:0.72(95\% Cl 0.62- 0.85) \\
\hline & & SRT $₫ 5 \rrbracket$ & & & \\
\hline & & ART $₫ 2 \bigotimes$ & 65625 & 7 years & RR:0.88(95\% Cl 0.64-1.20) \\
\hline & & SRT $₫ 5 \rrbracket$ & 195419 & 7 years & RR:0.94(95\% Cl 0.77-1.15) \\
\hline & & ART $₫ 2 \bigotimes$ & 268874 & 13 years & RR:0.77(95\% Cl 0.69-0.86) \\
\hline & & $\mathrm{SRT} \otimes 5 \square$ & & & \\
\hline & & ART®2】 & 74261 & 13 years & RR:0.94(95\% Cl 0.77-1.15) \\
\hline & & SRT $\otimes 5 \square$ & 194613 & 13 years & RR:0.70(95\% Cl 0.62-0.80) \\
\hline \multirow[t]{3}{*}{ Nelson (2016) } & $39-49$ & RCTs (9) & NR & 11.2 to 21.9 years & RR:0.92(95\% Cl 0.75-1.02) \\
\hline & $50-59$ & RCTs (7) & NR & 12.5 to 21.9 years & RR:0.86(95\% Cl 0.68-0.97) \\
\hline & $69-69$ & RCTs (5) & NR & 12.5 to 15.5 years & RR:0.67(95\% Cl 0.54-0.83) \\
\hline
\end{tabular}




\begin{tabular}{|llllll|} 
& $70-74$ & RCTs (3) & NR & 12.5 to 13.6 years & RR:0.80(95\% Cl 0.51-1.28) \\
Charles (1995) & $40-49$ & RCTs (7) & 128519 & 7 years & RR:0.76(95\% Cl 0.62-0.95) \\
\hline
\end{tabular}

RCT randomised controlled trial; CSS cross-sectional studies; ART adequately randomised trials; SRT suboptimally randomised trials

Table 3.

Main results of the included meta-analysis evaluating the accuracy of screening mammography

\begin{tabular}{|c|c|c|c|c|c|c|c|}
\hline No.study & Intervention & $\begin{array}{l}\text { Sensitivity } \\
(95 \% \mathrm{Cl})\end{array}$ & $\begin{array}{l}\text { Specificity } \\
(95 \% \mathrm{Cl})\end{array}$ & $\begin{array}{l}\text { AUC } \\
(95 \% \mathrm{Cl})\end{array}$ & $\begin{array}{l}\text { PLR } \\
(95 \% \mathrm{Cl})\end{array}$ & $\begin{array}{l}\text { NLR } \\
(95 \% \mathrm{Cl})\end{array}$ & $\begin{array}{l}\text { DOR } \\
(95 \% \mathrm{Cl})\end{array}$ \\
\hline Zhu(2018) & CESM & $89 \%(88 \%, 91 \%)$ & $84 \%(82 \%, 85 \%)$ & $0.96(0.94,0.98)$ & $3.73(2.68,5.2)$ & $0.1(0.06,0.15)$ & $71.36(36.28,140.39)$ \\
\hline \multirow[t]{2}{*}{ Hodgson(2016) } & DBT + FFDM & $\begin{array}{l}90.77 \%(80.7 \%, \\
96.51 \%)\end{array}$ & $\begin{array}{l}96.49 \% \\
(96.04 \%, 96.90 \%)\end{array}$ & NR & NR & NR & NR \\
\hline & FFDM & $\begin{array}{l}60.00 \% \\
(47.10 \%, 71.96 \%)\end{array}$ & $\begin{array}{l}95.55 \% \\
(95.04 \%, 96.01 \%)\end{array}$ & NR & NR & NR & NR \\
\hline \multirow[t]{2}{*}{ Souza(2013) } & $\mathrm{FM}$ & NR & NR & $\begin{array}{l}0.92(0.91 \\
0.92)\end{array}$ & NR & NR & NR \\
\hline & FFDM & NR & NR & $\begin{array}{l}0.91 \\
(0.89,0.93)\end{array}$ & NR & NR & NR \\
\hline \multirow[t]{2}{*}{ Song(2019) } & DM & $76 \%(70 \%, 81 \%)$ & $96 \%(94 \%, 97 \%)$ & $0.94(0.92,0.96)$ & $18.0(12.2,26.7)$ & $0.25(0.20,0.32)$ & $72(44,118)$ \\
\hline & $\mathrm{FM}$ & $76 \%(70 \%, 81 \%)$ & $97 \%(94 \%, 98 \%)$ & $0.92(0.89,0.94)$ & $22.5(13.1,38.8)$ & $0.25(0.20,0.31)$ & $91(52,157)$ \\
\hline Song(2019) & Mammography & $\begin{array}{l}85.7 \% \\
(77.7 \%-92.2 \%)\end{array}$ & $\begin{array}{l}95.3 \%(\mathrm{Cl} 95 \% \\
94.4 \%-96.1 \%)\end{array}$ & NR & NR & NR & NR \\
\hline Phi (2017) & Mammography & $55 \%(41 \%-69 \%)$ & $94 \%(90 \%-96 \%)$ & NR & NR & NR & NR \\
\hline
\end{tabular}

AUC area under curve; PLR Positive likelihood Ratio; NLR Negative likelihood ratio; DOR diagnostic odds ratio.

Table 4.

Main results of the included meta-analysis evaluating the recall rate and CDR of screening mammography

\begin{tabular}{|c|c|c|c|c|c|c|c|c|}
\hline \multirow[t]{3}{*}{ No.study } & \multirow{3}{*}{$\begin{array}{l}\text { Intervention } \\
\text { (A) }\end{array}$} & \multirow{3}{*}{$\begin{array}{l}\text { Intervention } \\
\text { (B) }\end{array}$} & \multirow[t]{3}{*}{$\mathbf{N}$} & \multicolumn{3}{|l|}{ Recall rate } & \multicolumn{2}{|l|}{ CDR } \\
\hline & & & & Recall rate & Recall rate & $\mathrm{ES}(95 \% \mathrm{Cl})$ & $\operatorname{CDR}(A)$ & CDR ( \\
\hline & & & & (A) & (B) & (A VS B) & & \\
\hline $\operatorname{lared}(2011)$ & DM & FM & 11 & $10497 / 190322$ & $26745 / 638348$ & RR: $1.07(0.94,1.22)$ & $1024 / 190,322$ & $3599 /$ \\
\hline Farber(2020) & DM & FM & 24 & $119638 / 3867923$ & $261652 / 8110599$ & $\begin{array}{l}\text { RD: } 0.00695(0.00347 \\
0.01042)\end{array}$ & $31015 / 5614900$ & $5621 \varepsilon$ \\
\hline Vinnicombe(2009) & FM & FFDM & 8 & $1404 / 31720$ & $406 / 8478$ & RR: 0.95(0.71,1.26) & $205 / 31720$ & $58 / 84$ \\
\hline Marinovich(2018) & DBT & $\mathrm{DM}$ & 17 & $31337 / 381424$ & $69626 / 678826$ & RD:-0.0219(-0.0298,-0.014) & $2093 / 350810$ & $2939 /$ \\
\hline \multirow[t]{2}{*}{ Giampietro(2020) } & $\mathrm{DBT}+\mathrm{DM}$ & DM & 13 & $25834 / 341780$ & $29715 / 437716$ & RR: $1.36(1.18,1.58)$ & $474 / 48482$ & $347 / 4$ \\
\hline & $\mathrm{DBT}+\mathrm{SM}$ & $\mathrm{DM}$ & 6 & $3196 / 94578$ & $3905 / 119359$ & RR: $1.08(0.92, .26)$ & $754 / 94568$ & $702 / 1$ \\
\hline Phi(2018) & $\mathrm{DBT}+\mathrm{DM}$ & $\mathrm{DM}$ & 7 & $12705 / 12221$ & $27808 / 226967$ & RR: $0.72(0.64,0.8)$ & 704/115838 & $854 / 1$ \\
\hline Yun(2017) & $\begin{array}{l}\text { DBT + } \\
\text { FFDM }\end{array}$ & FFDM & 11 & $743 / 112624$ & $970 / 212917$ & RR: $1.29(1.164,1.429)$ & NA & NA \\
\hline
\end{tabular}

ES effect size; CDR, cancer detection rate.

\section{Figures}




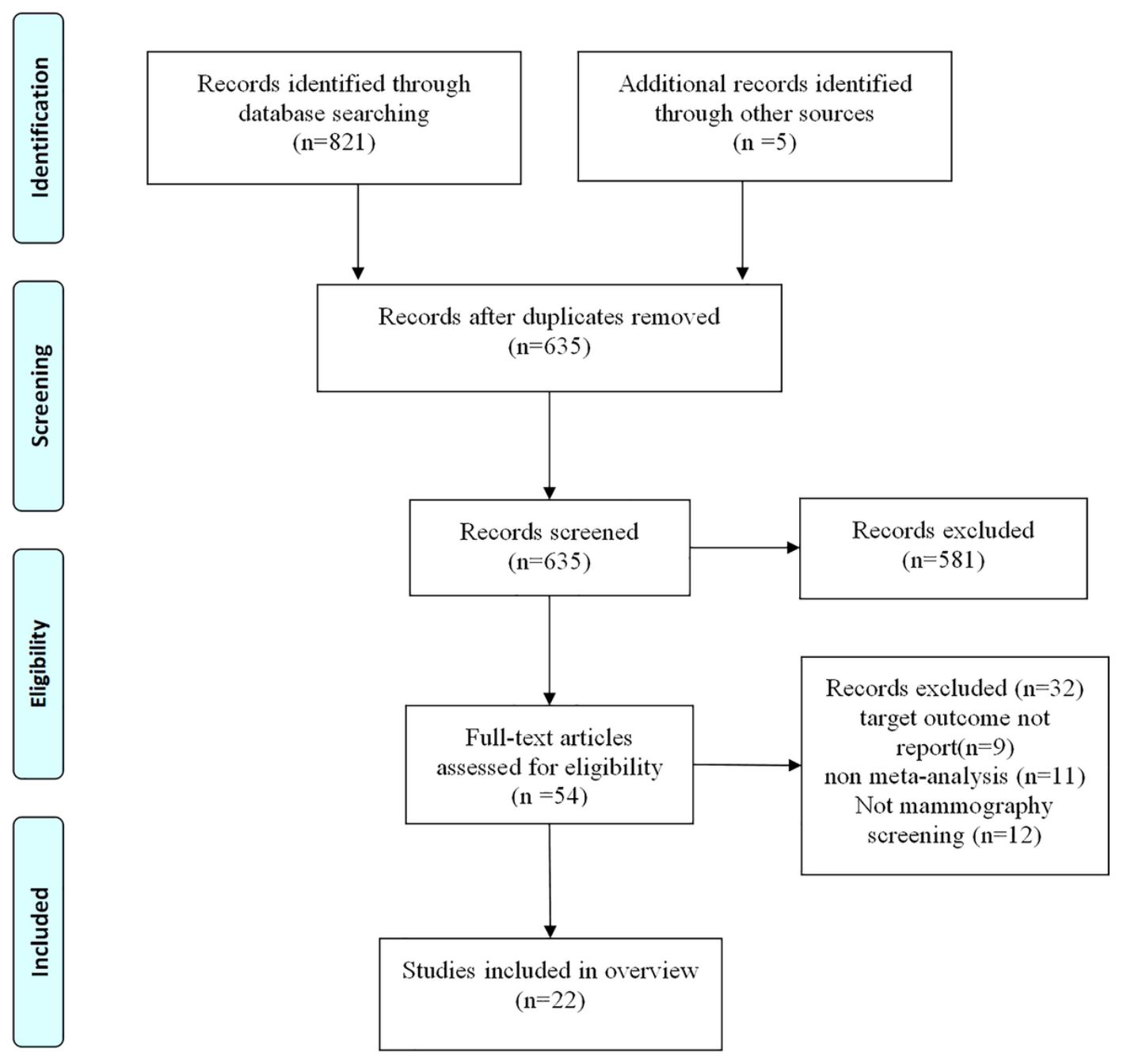

Figure 1

Flow diagram of the study selection. 


\begin{tabular}{|c|c|c|c|c|c|}
\hline \multirow[b]{2}{*}{ Review } & \multirow[b]{2}{*}{$\begin{array}{l}\text { 1. Study eligibility } \\
\text { criteria }\end{array}$} & \multicolumn{2}{|c|}{ Phase 2 } & \multirow[b]{2}{*}{$\begin{array}{l}\text { 4. Synthesis and } \\
\text { findings }\end{array}$} & \multirow{2}{*}{$\begin{array}{c}\text { Phase } 3 \\
\text { Risk of bias } \\
\text { in the review }\end{array}$} \\
\hline & & $\begin{array}{c}\text { 2. Identification } \\
\text { and selection studies }\end{array}$ & $\begin{array}{c}\text { 3. Data collection } \\
\text { and study appraisal }\end{array}$ & & \\
\hline $\begin{array}{l}\text { Zhu(2018) } \\
\end{array}$ & (2) & (2) & () & (2) & (2) \\
\hline Iared(2011) & (2) & (2) & (2) & (2) & (2) \\
\hline Sarah(2009) & (2) & (2) & (2) & (2) & (2) \\
\hline Giampietro(2020) & (1) & (-) & () & () & () \\
\hline Hodgson(2016) & ()ㅜ & ()ㅜㅇ & () & () & (2) \\
\hline Souza(2013) & () & 숭 & ○) & $?$ & (2) \\
\hline Yun(2017) & (2) & (2) & (-) & (2) & (2) \\
\hline Song(2019) & (2) & (2) & () & (2) & (2) \\
\hline Marinovich(2018) & () & (2) & () & (2) & (2) \\
\hline Phi(2018) & (2) & (2) & (-) & () & (2) \\
\hline Farber(2020) & () & (2) & (2) & () & (2) \\
\hline Nickson(2012) & (2) & (2) & (2) & (2) & (2) \\
\hline Magnus(2011) & (2) & (2) & (2) & (2) & (2) \\
\hline Gotzsche(2000) & (2) & ๑ & $?$ & (2) & (2) \\
\hline Hendrick(1997) & (2) & $?$ & $?$ & (2) & (2) \\
\hline Kerlikowske(1995) & (2) & (2) & $?$ & (2) & (2) \\
\hline Jacklyn(2016) & (2) & (2) & (2) & (2) & (2) \\
\hline Posso(2017) & () & (2) & $?$ & (웅 & (2) \\
\hline Phi (2017) & (2) & (2) & (2) & $?$ & (2) \\
\hline Gotzsche (2013) & () & (1) & () & () & () \\
\hline Nelson (2016) & (2) & (2) & () & () & (2) \\
\hline Charles (1995 & (2) & (2) & (2) & (2) & (2) \\
\hline
\end{tabular}

(i) = low risk; $: 2$ = high risk; $?=$ unclear risk

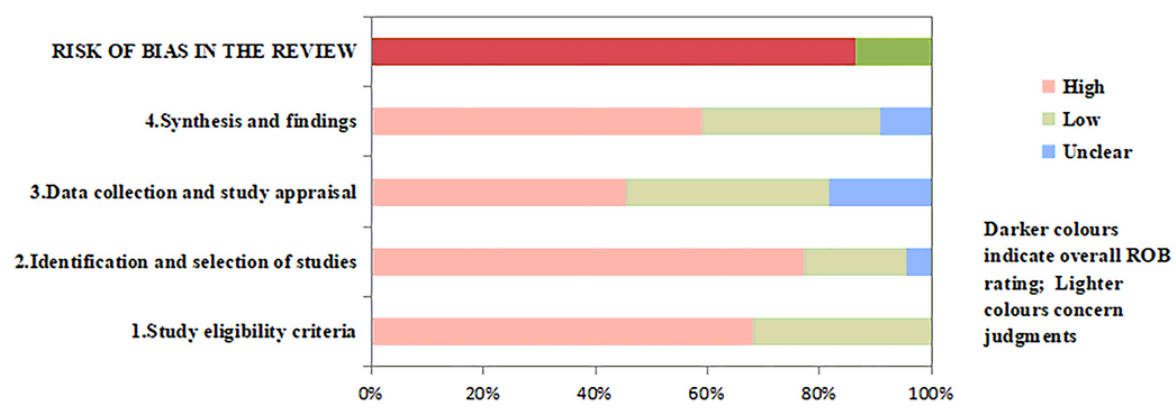

Figure 2

ROBIS result of 22 included studies.

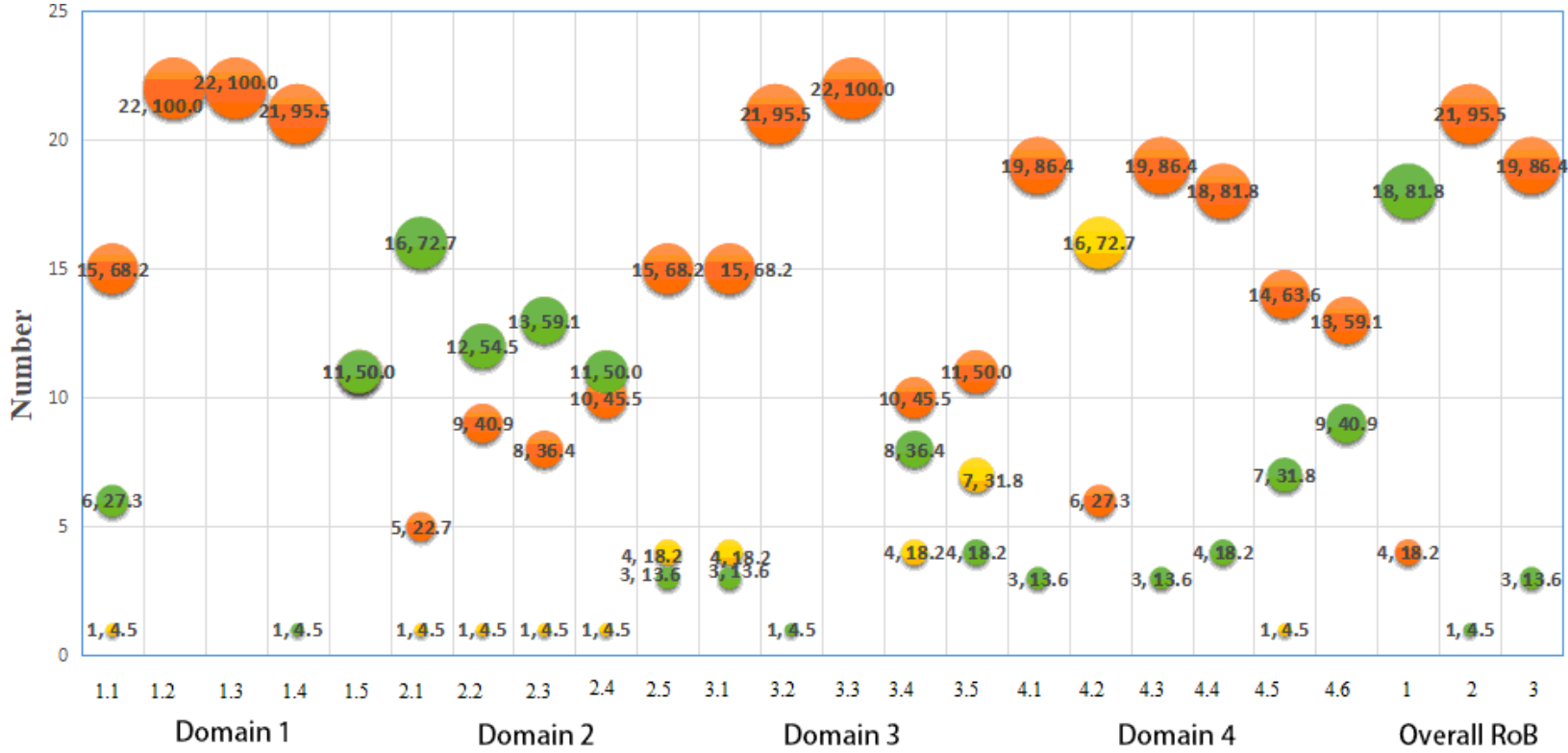

- Yes/Probably Yes Partial No/Probably No

\section{Figure 3}

The full compliance rate of each ROBIS item. 


\section{Supplementary Files}

This is a list of supplementary files associated with this preprint. Click to download.

- Table1.Maincharacteristicsofinludedstudies.docx

- PRISMADTAChecklist.doc

- S1Searchstrategy.docx 